\title{
Characterisation of the Surface Chemistry of Activated Carbon by Molecular Simulation of Water Adsorption
}

\author{
M. Jorge and N. A. Seaton* \\ School of Chemical Engineering, University of Edinburgh \\ King's Buildings, Mayfield Road, Edinburgh, EH9 3JL, UK \\ Tel.: (+44) 131650 4867; Fax: (+44) 1316506551 \\ e-mail: n.seaton@ed.ac.uk
}

We propose a model for activated carbon incorporating both structural and chemical heterogeneity. Structural heterogeneity is represented by an array of slit-shaped pores, characterised by a pore size distribution. This distribution was calculated from experimental data on pure-component adsorption of ethane, using Grand Canonical Monte Carlo simulations to describe adsorption at the individual pore level. Chemical heterogeneity is represented in our model by including oxygen-containing surface sites. The results from molecular simulation in the model carbon are compared to the experimental pure-water adsorption isotherm on the same sample. From such a comparison, one is able to draw conclusions regarding the concentration and distribution of surface sites in pores of different width.

\section{INTRODUCTION}

Activated carbons are extensively used for adsorption processes in industrial applications. A number of these applications (such as air purification) involves the separation of organic molecules from mixtures containing trace amounts of water. For an efficient design of these processes, it is essential to develop a model for the activated carbon adsorbents that possesses predictive capabilities. Such a model must take into account the fundamental characteristics of the adsorbent that affect the adsorption of the molecular species of interest. Activated carbons are amorphous materials composed of graphite plates stacked together in a more or less irregular way [1]. Adsorption occurs mainly in the interstices of the plates. As a consequence of the activation process, chemically heterogeneous groups of several types can be present on the edges of the graphitic plates or on defect sites. The most common types are oxygen-containing groups, such as carboxyl, carbonyl and hydroxyl [2]. Structural heterogeneity has a strong effect on adsorption of all chemical species, while chemical heterogeneity affects mainly the adsorption of polar species. In the case of water/organic mixtures, both types of heterogeneity must be taken into account for the development of a suitable model.

The most widely used model of the structure of activated carbon is an array of slitshaped pores, characterised by a pore size distribution (PSD). This approach has been used successfully to model the adsorption of non-polar species [see, e.g. 3-6]. Although the PSD model neglects a number of aspects of the carbon structure, such as network connectivity and surface defects, the more complex models proposed (see e.g. $[7,8]$ ) have not yet advanced to the stage where they can quantitatively match adsorption in real solids, even of non-polar compounds. Attempts to include chemical heterogeneity in model carbons have not been so 
prolific. To our knowledge, the first of these attempts was undertaken by Segarra and Glandt [9]. Their model consisted of a disordered stacking of disk-shaped graphitic platelets. Surface polarity was represented by dipole moments smeared along the edges of the plates. Agreement between the model and experimental data was only qualitative. A later model, by McCallum et al. [10], included a representation of the discrete nature of the surface sites. The basis of the model was a PSD, determined by density functional theory and nitrogen adsorption, and surface sites were randomly distributed on the surface of the pores. Water molecules and surface oxygen atoms were represented by simplified molecular models, based on square-well potentials to mimic hydrogen bonding. The total concentration of active sites was determined experimentally by titration methods and the interaction potential between water molecules and surface sites was obtained from a fit to low-pressure water adsorption data. The simulations were performed in only a few pore widths and then interpolated, using the semiempirical equation of Talu and Meunier [11], to represent the continuous PSD. Although agreement with experiment was reasonable, the potential models ignore the longrange nature of the electrostatic interactions among water molecules, and between the water molecules and polar surface groups. This makes it difficult to extent the model to more general cases (e.g. other temperatures, multicomponent adsorption). The most recent model of activated carbon to include chemical heterogeneity was proposed by Brennan et al. [12]. In this model, the structure of the adsorbent is represented by a stacking of graphitic plates of several sizes, which is optimised to reproduce experimentally determined carbon-carbon radial distribution functions by a procedure termed reverse Monte Carlo simulation [7]. Polar carbonyl sites were placed randomly on the edges of the plates. Although it is a more realistic representation of activated carbon, this model is also extremely complex, which carries disadvantages such as very computationally demanding simulations and difficulty of application to a wide range of adsorbents. No comparison of this model with experiment has yet been presented.

In this paper, we present a model for activated carbon that takes into account the characteristics of the adsorbent that affect the adsorption of both polar and non-polar species. The structure of the carbon is represented by a PSD, obtained from the analysis of pure-ethane adsorption, and chemical heterogeneity is included by placing regularly distributed carbonyl sites on the surface of the pores. The single-pore isotherms for water and ethane are calculated by grand canonical Monte Carlo (GCMC) simulation.

\section{GCMC SIMULATION IN SINGLE PORES}

This method is particularly suited for adsorption studies, since the temperature $(T)$, volume $(V)$ and chemical potential $(\mu)$ are kept constant, while the total number of molecules is allowed to fluctuate. By averaging the number of adsorbed molecules at equilibrium and calculating the pressure $(P)$ from the chemical potential, one can obtain adsorption isotherms in model pores. The Peng-Robinson equation of state [13] was used for the pressure calculations. We have used the general methodology for GCMC simulations $[14,15]$. For each

point of the isotherm the system was allowed to equilibrate for at least $10^{7}$ steps (each step consisting of a displacement, creation or destruction trial) and the sampling period was divided into 20 blocks of at least $10^{5}$ steps each.

Ethane molecules are represented by two Lennard-Jones (L-J) centres, one for each $\mathrm{CH}_{3}$ group. Geometrical and potential parameters were taken from Cracknell and Nicholson [16]. Water is represented by the SPC/E potential. This model is composed of a single L-J site, at a location corresponding to the oxygen atom in the water molecule, and three point charges, two positive ones located at the positions of the hydrogen atoms and a negative charge at the 
centre of the oxygen atom. Geometric and potential parameters were taken from Berendsen et al. [17]. The pores are slit-shaped, bounded by two parallel walls comprising an infinite number of graphite layers. Each layer is composed of L-J atoms, which are uniformly smeared out to form smooth walls. The potential between an L-J centre in an adsorbate molecule and each of the pore walls is given by the 10-4-3 potential of Steele [18]. The polar surface sites are carbonyl groups, represented by the OPLS potential for $\mathrm{C}=\mathrm{O}$ groups in aminoacids [19]. These include an L-J centre and a negative point charge for the oxygen atom and a positive point charge on the carbon atom in the basal plane of graphite, as described in a previous publication [20]. The simulation cell is rectangular, bounded in the $z$ direction by the pore walls and replicated in the $x$ and $y$ directions by using periodic boundary conditions. The length of the simulation cell in both directions parallel to the wall was chosen to be $3 \mathrm{~nm}$ since this was shown to be sufficient to eliminate any finite-size effects [21]. The calculation of the potential between two point charges requires the use of special techniques, due to its long-ranged character. We have compared several of these techniques for the slab geometry [22] and concluded that the method proposed by Heyes and van Swol [23] provided the best compromise between accuracy and speed of computation. This method performs a summation of the Coulomb potential over a small number of periodic boxes and then includes a correction term for the remaining boxes up to infinity. Cross-species Lennard-Jones parameters were calculated using the Lorentz-Berthelot combining rules.

\section{MODEL FOR ACTIVATED CARBON}

The structure of the activated carbon is represented by a distribution of slit-shaped pores. In a PSD model, the total adsorption is given by the adsorption integral equation (AIE) [4]:

$$
N(P)=\int_{w_{\min }}^{w_{\max }} f(w) \rho(P, w) d w
$$

where $N(P)$ is the total number of moles adsorbed at a pressure $P, w_{\min }$ and $w_{\max }$ are the widths of the smallest and largest pores present, $\rho(P, w)$ is the molar density of the adsorbent at a pressure $P$ in a pore of width $w$, and $f(w)$ is the PSD. In order to calculate the PSD of a given adsorbent, Eq. (1) must be solved for $f(w)$, which presents an ill-posed problem. To solve the AIE, we have used the method of Davies et al. [24]. Essentially, the PSD is discretised and a representative result is obtained by incorporating a regularisation factor in the minimisation procedure. The optimum factor is determined by an analysis of the error of the fit. This method has been successful in predicting pure-component and binary adsorption of methane and ethane in several activated carbons from molecular simulation [25].

We have calculated a PSD for BPL carbon based on the experimental ethane adsorption of Russell and LeVan [26] at three different temperatures (273, 298 and $323 \mathrm{~K})$. This set of experimental data was chosen since these workers present adsorption of pure water and pure ethane, as well as their mixtures, in BPL activated carbon over a wide range of temperatures. After the single pore isotherms for ethane were obtained from GCMC simulations, the AIE was solved using a non-linear minimisation routine [27] interfaced through the GAMS mathematical programming package [28]. The distribution with the optimum regularisation factor is shown in Figure 1 and the fit to the experimental data in Figure 2. The calculated PSD fits the experimental data well over the entire pressure range. 


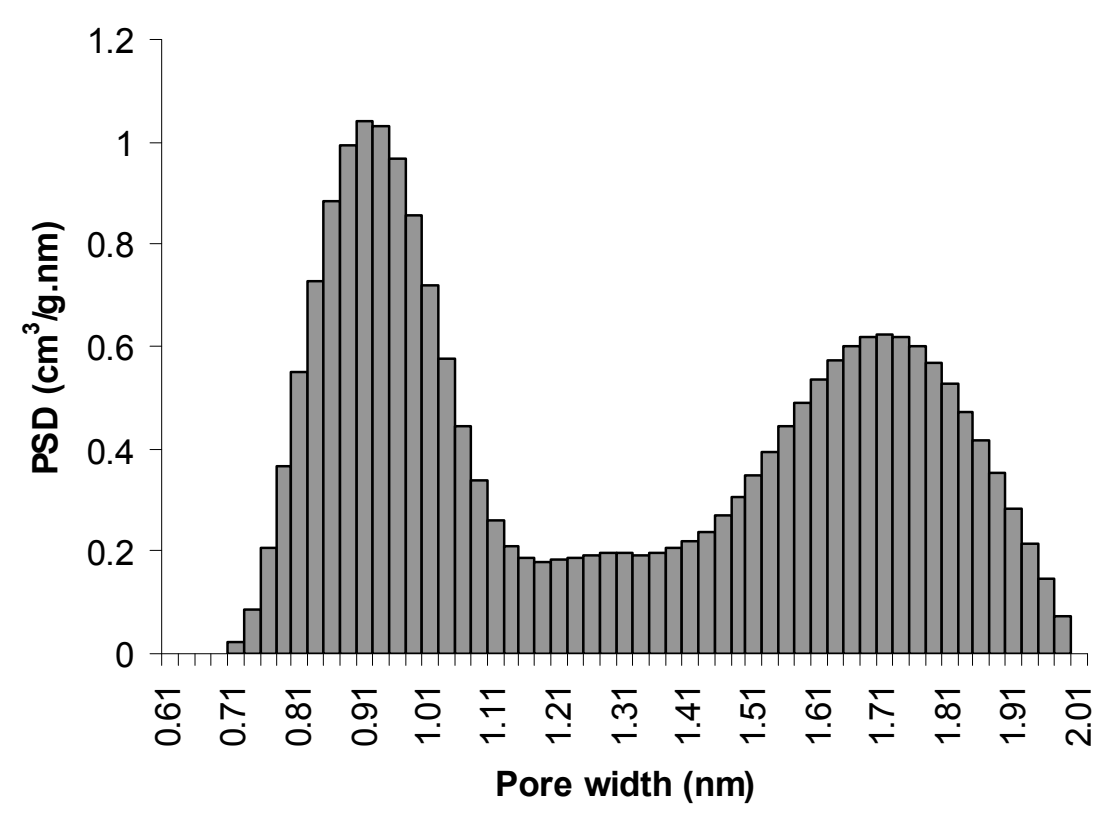

Figure 1: Pore size distribution of BPL activated carbon determined from the analysis of pure ethane adsorption at 273, 298 and $323 \mathrm{~K}$.

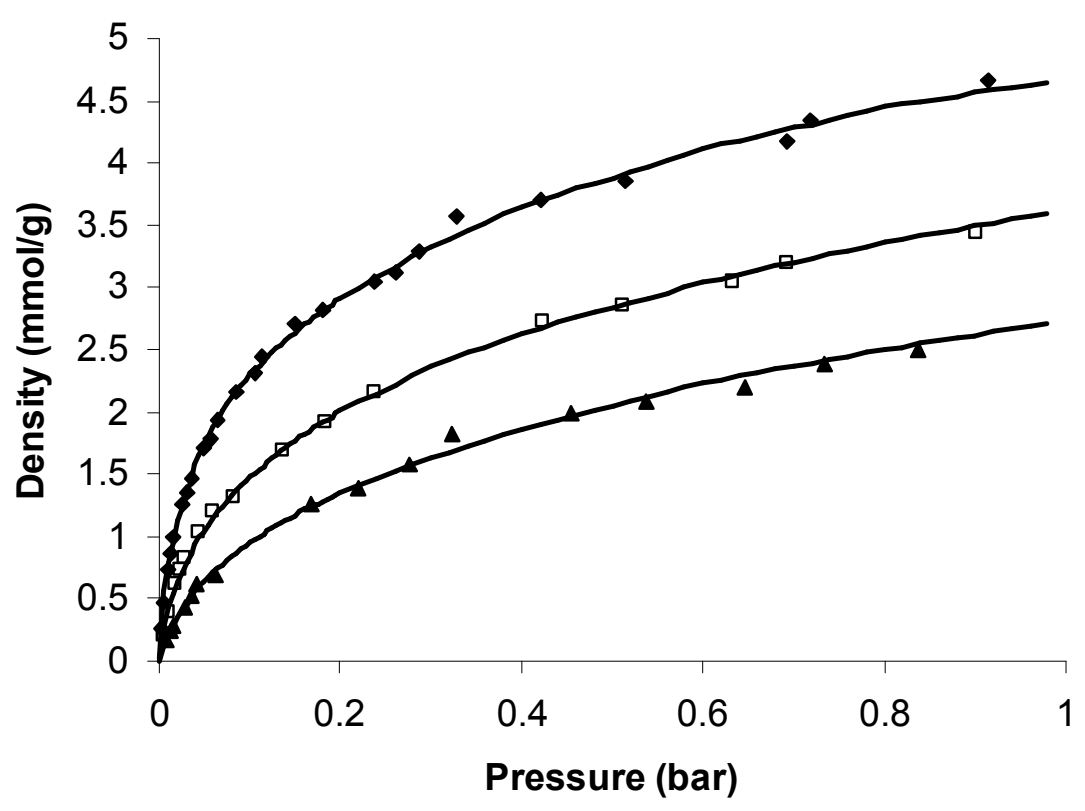

Figure 2: Fit to the pure ethane experimental data of Russell and LeVan [26] using the PSD of Figure 1. Diamonds are for data at $273 \mathrm{~K}$, open squares are for $298 \mathrm{~K}$ and triangles are for $323 \mathrm{~K}$.

After determining the structure of our activated carbon, we have incorporated a degree of chemical heterogeneity in the model by making some assumptions: the sites were all of the same type (carbonyl groups); they were regularly distributed on the surface of the carbon; and their concentration was considered to be the same in every pore, regardless of its width. The first assumption is justified on the basis that both experimental [29] and molecular simulation [20] results suggest that the amount of water adsorbed in activated carbon depends on the 
concentration of oxygen on the surface, regardless of its functionality. As for the distribution of the polar sites on the surface, we have observed from molecular simulation that the local density of active sites has a strong impact on low-pressure adsorption but almost no effect on capillary condensation [20]. Since most of the experimental water adsorption data was obtained at high pressure, in the region of condensation (see Figure 4), the assumption of a regular distribution of isolated sites will have little effect on the comparison. Finally, the concentration of surface sites was set to be the same in pores of all sizes, in the absence of $a$ priori information to the contrary.

These assumptions reduce the process of the characterisation of the chemical heterogeneity to the determination of a single variable - the overall density of carbonyl sites in the carbon. This concentration was estimated by analysing the experimental pure water adsorption isotherm at $298 \mathrm{~K}$ as follows. First the modal pore width was determined based on the PSD obtained from pure ethane adsorption. Then a series of GCMC simulations in a pore of that width $(1.24 \mathrm{~nm})$ for different concentrations of active sites was performed. The chosen concentration was such that the pressure of spontaneous condensation in the single-pore isotherm corresponded roughly to the inflexion point of the experimental isotherm. After this procedure, GCMC isotherms for 13 pore widths containing the chosen concentration of active sites $\left(1.33\right.$ sites $\left./ \mathrm{nm}^{2}\right)$ were calculated. These pore widths span the whole range of the PSD of Figure 1 and retain the characteristics of the distribution. Some of these isotherms are shown in Figure 3.

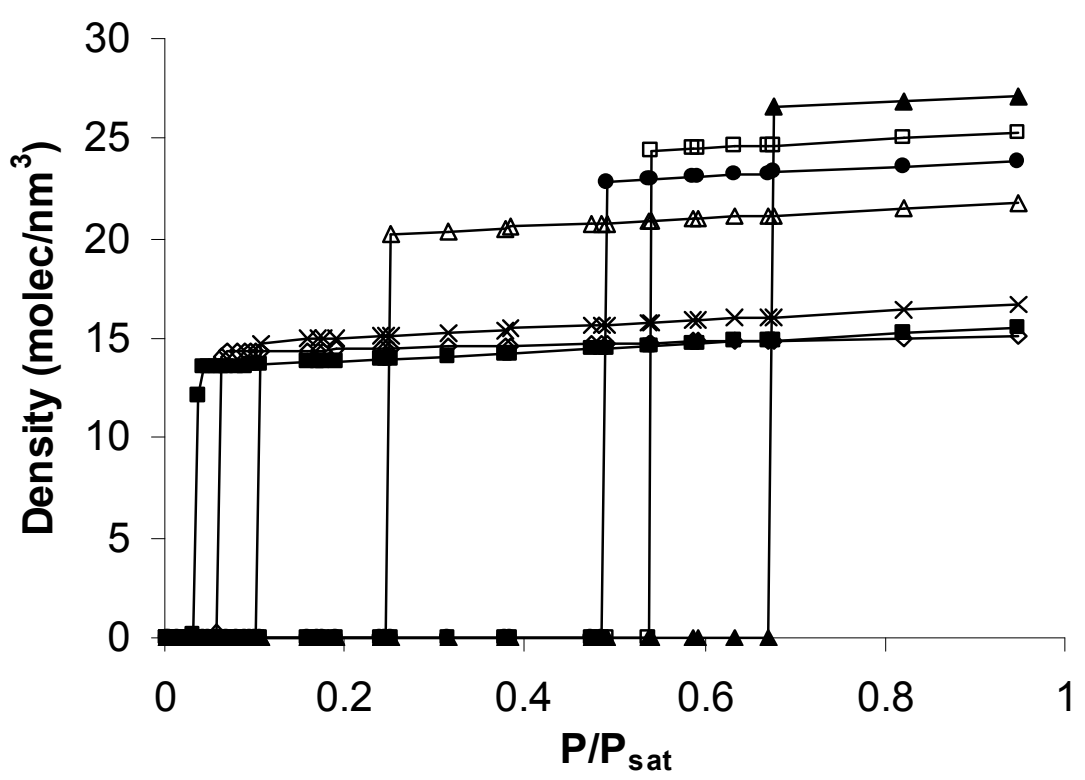

Figure 3: GCMC adsorption isotherms for pure water at $298 \mathrm{~K}$ in slit-shaped pores with a concentration of surface sites of 1.33 sites $/ \mathrm{nm}^{2}$ and different widths: filled squares $-0.68 \mathrm{~nm}$; open diamonds $-0.76 \mathrm{~nm}$; crosses $-0.84 \mathrm{~nm}$; open triangles $-1.04 \mathrm{~nm}$; filled circles $-1.24 \mathrm{~nm}$; open squares $-1.44 \mathrm{~nm}$; filled triangles $-2.04 \mathrm{~nm}$. The pressure is reduced by the saturation pressure at the same temperature $(0.03167$ bar $)$.

At low pressure, there is hardly any water adsorbed in the pores. At a certain characteristic pressure (specific to each pore width), there is a step-like increase in the amount adsorbed, until the pores are completely filled. All the isotherms, down to the smallest pore width, exhibit this discontinuous transition, which is evidence of capillary condensation. It is 
worth noting that the pressure of spontaneous condensation in a GCMC simulation does not represent the equilibrium vapour-liquid transition pressure in the pore. Rather, during adsorption calculations, the vapour-like phase remains metastable up to pressures well above equilibrium [20] (the inverse is true during desorption runs). The fact that GCMC isotherms are used in our calculations is justified by the fact that metastability is also expected to occur in porous solids during adsorption experiments (see e.g. [30,31]). Even though the two types of metastability (simulated and experimental) are distinct, and represent different processes [31,32], recent studies in well characterised porous materials [33] show good agreement between simulation and experiment and suggest that the experimental adsorption branch is closer to the point of spontaneous condensation in GCMC simulation than to the equilibrium transition. It is worth noting that we have recently calculated the equilibrium phase transition pressure of water in both graphitic [21] and activated [20] carbon pores by molecular simulation using the gauge-cell method [34]. Our results confirm the occurrence of capillary condensation even in very small pores, as well as the presence of metastable vapour and liquid phases during GCMC adsorption and desorption runs, respectively.

Integration of the single-pore isotherms over the PSD produced the total adsorption isotherm for the model carbon, which can be compared with the experimental data. This comparison is shown in Figure 4.

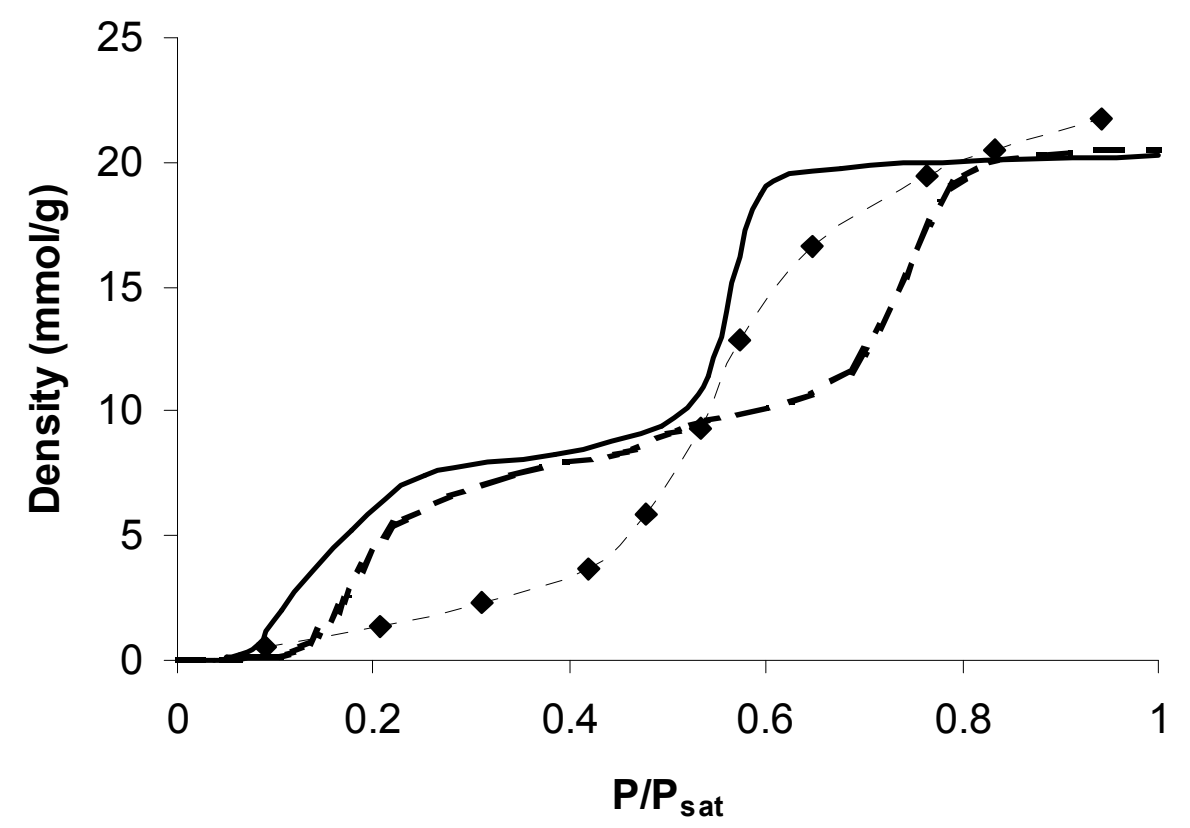

Figure 4: Comparison between the experimental data of Russell and LeVan [26] for pure water at 298 $\mathrm{K}$ (diamonds) and the isotherms obtained from the model carbon at two different concentrations of carbonyl sites: 1.333 sites $/ \mathrm{nm}^{2}$ (solid line) and 0.889 sites $/ \mathrm{nm}^{2}$ (dashed line). The dotted line connecting the experimental data points is a guide to the eye.

As we can see from Figure 4, the simulated isotherm (solid line) is qualitatively different from the experimental isotherm (dotted line). The bimodal character of the PSD is reflected in the simulated isotherm, producing two "steps". On the other hand, the experimental isotherm has a typical S shape. Furthermore, the simulation results lie almost entirely at lower pressures than the experiment, which suggests that the concentration of surface sites is too high. As an attempt to improve the model, we have performed simulations 
for a surface site concentration of 0.889 sites $/ \mathrm{nm}^{2}$. The results are shown as the dashed line in Figure 4. The improvement in the agreement with experiment is only slight, as the simulated isotherm still exhibits two steps. Any other concentration of surface sites would shift the isotherm on the pressure axis, but would retain the bimodal character of the isotherm. The choice of a different local distribution of sites would have a significant impact on adsorption at low pressure, but not on the condensation pressure [20]. Thus, we can conclude that our model, in which the concentration of surface sites is the same for pores of all sizes, is incapable of matching the experimental data for water. (The excellent fit of the model to ethane adsorption over a wide range of temperature and pressure demonstrates that the description of structural heterogeneity by a PSD is sound.)

It follows that a possible improvement to the model would be to allow the concentration of active sites to differ from pore width to pore width. A model in which the pores of width above $1.24 \mathrm{~nm}$ contain a slightly higher amount of active sites and those between 0.84 and $1.24 \mathrm{~nm}$ are less activated would result in a closer match with the experimental isotherm. Regardless of this fact, the overall concentration of oxygen on the surface of the carbon is likely to be of around 0.889 sites $/ \mathrm{nm}^{2}$. By using the PSD and the geometrical definition of the model pores, we are able to calculate a value for the surface area of our model carbon. This value was $925 \mathrm{~m}^{2} / \mathrm{g}$, which compares well with the BET surface area of $1200 \mathrm{~m}^{2} / \mathrm{g}$ determined experimentally on the same sample [3] (if one bears in mind that the BET method is likely to overestimate the real specific area of the carbon, since it does not take into account the enhancement of the adsorption energy in small pores). By using this value, one is able to determine the concentration of oxygen per gram of carbon. This value, $1.365 \mathrm{mmol} / \mathrm{g}$, is within the range of $1.14-1.40 \mathrm{mmol} / \mathrm{g}$ determined experimentally by MacDonald and coworkers using temperature-programmed desorption on BPL carbon [35-37].

\section{CONCLUSIONS}

In this paper we have presented preliminary results of an attempt to model both structural and chemical heterogeneity of activated carbon adsorbents, in order to quantitatively predict water adsorption. We have represented the structure of the carbon by a distribution of smooth slit-shaped pores, with regularly distributed polar sites as a measure of chemical heterogeneity. The concentration of surface oxygen that provides the best agreement with the experimental data is within the range of experimentally determined values. From a comparison between simulation and experiment we are able to draw conclusions with respect to the distribution of surface sites along pores of different width. Thus we have observed that the small micropores in BPL carbon are likely to exhibit a lower concentration of sites than the larger pores. An attempt to improve our model by simulating adsorption in pores with different concentrations of surface sites is underway. The predictive capabilities of the model will be assessed based on a comparison with experimental binary adsorption of water and ethane.

\section{ACKNOWLEDGEMENTS}

M. Jorge wishes to acknowledge financial support from Fundação para a Ciência e Tecnologia - Programa PRAXIS XXI.

\section{REFERENCES}

1. B. McEnaney, Carbon, 26 (1988), 267. 
2. H. P. Boehm, Carbon, 32 (1994), 759.

3. B. P. Russell and M. D. LeVan, Carbon, 32 (1994), 845.

4. N. A. Seaton, J. P. R. B. Walton and N. Quirke, Carbon, 27 (1989), 853.

5. C. Nguyen and D. D. Do, Langmuir, 16 (2000), 1319.

6. P. I. Ravikovitch, A. Vishnyakov, R. Russo and A. V. Neimark, Langmuir, 16 (2000), 2311.

7. K. T. Thomson and K. E. Gubbins, Langmuir, 16 (2000), 5761.

8. J. R. Dahn, W. Xing and Y. Gao, Carbon, 35 (1997), 825.

9. E. I. Segarra and E. D. Glandt, Chem. Eng. Sci, 49 (1994), 2953.

10. C. L. McCallum, T. J. Bandosz, S. C. McGrother, E. A. Müller and K. E. Gubbins, Langmuir, 15 (1999), 533.

11. O. Talu and F. Meunier, AIChE J., 42 (1996), 809.

12. J. K. Brennan, T. J. Bandosz, K. T. Thomson and K. E. Gubbins, Colloids and Surfaces A: Physicochem. Eng. Aspects, 187-188 (2001), 539.

13. S. I. Sandler, Chemical and Engineering Thermodynamics, Wiley, New York, 1989.

14. M. P. Allen and D. J. Tildesley, Computer Simulation of Liquids, Clarendon Press, Oxford, 1989.

15. D. Frenkel and B. Smit, Understanding Molecular Simulation, Academic Press, London, 1996.

16. R. F. Cracknell and D. Nicholson, J. Chem. Soc., Faraday Trans., 90 (1994), 1487.

17. H. J. C. Berendsen, J. R. Grigera and T. P. Straatsma, J. Phys. Chem., 91 (1987), 6269.

18. W. A. Steele, The Interaction of Gases with Solid Surfaces, Pergamon Press, Oxford, 1974.

19. W. L. Jorgensen and J. Tirado-Rives, J. Am. Chem. Soc., 110 (1988), 1657.

20. M. Jorge, C. Schumacher and N. A. Seaton, submitted to Langmuir.

21. M. Jorge and N. A. Seaton, submitted to Mol. Phys..

22. M. Jorge and N. A. Seaton, Mol. Phys., 100 (2002), 2017.

23. D. M. Heyes and F. van Swol, J. Chem. Phys., 75 (1981), 5051.

24. G. M. Davies, N. A. Seaton and V. S. Vassiliadis, Langmuir, 15 (1999), 8235.

25. G. M. Davies and N. A. Seaton, Langmuir, 15 (1999), 6263.

26. B. P. Russell and M. D. LeVan, Ind. Eng. Chem. Res., 36 (1997), 2380.

27. MINOS 5.0, Systems Optimization Laboratory, Department of Operations Research, Stanford University, CA, 1988.

28. GAMS Development Corporation, 1217 Potomac Street, NW, Washington, DC 20007, 1998.

29. S. S. Barton, M. J. B. Evans and J. A. F. MacDonald, Langmuir, 10 (1994), 4250.

30. G. S. Heffelfinger, F. van Swol and K. E. Gubbins, J. Chem. Phys., 89 (1988), 5202.

31. L. Sarkisov and P. A. Monson, Langmuir, 16 (2000), 9857.

32. M. Schoen, C. L. Rhykerd, J. H. Cushman and D. J. Diestler, Mol. Phys., 66 (1989), 1171

33. P. I. Ravikovitch, A. Vishnyakov and A. V. Neimark, Phys. Rev. E, 64 (2001), 011602.

34. A. V. Neimark and A. Vishnyakov, Phys. Rev. E, 62 (2000), 4611.

35. S. S. Barton, M. J. B. Evans, S. Liang and J. A. F. MacDonald, Carbon, 34 (1996), 975.

36. S. S. Barton, M. J. B. Evans, E. Halliop and J. A. F. MacDonald, Carbon, 35 (1997), 1361.

37. J. A. F. MacDonald, M. J. B. Evans, S. Liang, S. E. Meech, P. R. Norman and L. Pears, Carbon, 38 (2000), 1825. 